\title{
PROBING THE NATURE OF THE MASSIVE BLACK HOLE BINARY CANDIDATE SDSS J1536+0441*
}

\author{
R. Decarli ${ }^{1}$, M. Dotti ${ }^{2}$, R. Falomo ${ }^{3}$, A. Treves $^{1}$, M. Colpi ${ }^{4}$, J. K. Kotilainen $^{5}$, C. Montuori $^{4}$, And M. Uslenghi $^{6}$ \\ ${ }^{1}$ Dipartimento di Fisica e Matematica, Università dell'Insubria, via Valleggio 11, I-22100 Como, Italy; roberto.decarli@mib.infn.it \\ ${ }_{2}^{2}$ Department of Astronomy, University of Michigan, Ann Arbor, MI 48109, USA \\ ${ }^{3}$ INAF-Osservatorio Astronomico di Padova, Vicolo dell'Osservatorio 5, I-35122 Padova, Italy \\ ${ }^{4}$ Dipartimento di Fisica, Università degli Studi di Milano-Bicocca, piazza delle Scienze 3, 20126 Milano, Italy \\ ${ }^{5}$ Tuorla Observatory, Department of Physics and Astronomy, University of Turku, Väisäläntie 20, FI-21500 Piikkiö, Finland \\ ${ }^{6}$ INAF-IASF Milano, Via E. Bassini 15, Milano I-20133, Italy \\ Received 2009 June 12; accepted 2009 July 29; published 2009 September 4
}

\begin{abstract}
We present an imaging study of the black hole binary candidate SDSS J1536+0441 $(z \approx 0.3893)$, based on deep, high-resolution $V_{z} K$ images collected at the ESO/Very Large Telescope. The images clearly show an asymmetric elongation, indicating the presence of a companion source at $\sim 1^{\prime \prime}(\approx 5 \mathrm{kpc}$ projected distance) east from the quasar. The host galaxy of the quasar is marginally resolved. We find that the companion source is a luminous galaxy, the light profile of which suggests the presence of an unresolved, faint nucleus (either an obscured active galactic nucleus or a compact stellar bulge). The study of the environment around the quasar indicates the occurrence of a significant overdensity of galaxies with a redshift compatible with $z \approx 0.4$. This suggests that it resides in a moderately rich cluster of galaxies.
\end{abstract}

Key words: quasars: individual (SDSS J153636.22+044127.0)

\section{INTRODUCTION}

Boroson \& Lauer (2009) discovered peculiar features in the optical spectrum of SDSS J153636.22+044127.0 (hereafter SDSS J1536+0441), a $z=0.3893$ radio-quiet quasar. Three line systems are found at different redshifts: a set of broad and narrow emission lines at $z=0.3893$, a set of only broad emission lines at $z=0.3727$, and a third set of very narrow absorption lines at an intermediate redshift $(z=0.3878)$. Boroson \& Lauer (2009) interpreted the former two systems in terms of the broad line region emission around two massive black holes within the same galaxy, constituting a massive black hole binary (BHB) with subparsec separation.

Chornock et al. (2009a, 2009b) questioned the BHB scenario, ascribing the peculiar spectroscopic features of the quasar to a single active massive black hole with a particular disk-like geometry of the broad line region, similar to Arp 102B. This scenario is also supported by the detection of a bump in the red wings of broad emission line profiles, at $z \approx 0.4$.

Motivated by the claim by Boroson \& Lauer (2009), Wrobel \& Laor (2009) observed SDSS J1536+0441 using the VLA, discovering two radio sources (VLA-A and VLA-B) separated by $0^{\prime} \cdot 97$ within the quasar optical localization region. Independently, NIR observations led by our group (Decarli et al. $2009 \mathrm{~b}$ ) revealed the occurrence of a companion galaxy of the quasar, located in correspondence with VLA-B. This was subsequently confirmed in the optical wavelengths by Lauer \& Boroson (2009) using the Hubble Space Telescope.

Wrobel \& Laor (2009) and Decarli et al. (2009b) suggested that each nucleus of the two galaxies is related to a single set of broad emission lines. The validity of this picture can be tested with high angular resolution spectroscopy, in order to prove if the two sets of broad emission lines are spatially separated. Lauer \& Boroson (2009) and Chornock et al. (2009b) presented optical spectra of SDSS J1536+0441. Both groups suggest that the regions responsible for the emission of the two sets of broad

\footnotetext{
* Based on observations collected at the European Organisation for Astronomical Research in the Southern Hemisphere, Chile (DDT programme: 282.B-5059).
}

lines are spatially coincident. Nevertheless, both observations were carried out under non-optimal seeing conditions (larger than the angular separation of the two objects along the slit), hindering a clear comprehension of the contribution of the active nucleus in VLA-B to the spectrum of SDSS J1536+0441.

The scenario of a superposition of two active galactic nuclei (AGNs) has already been invoked by Heckman et al. (2009) to explain the spectral properties of another BHB candidate (SDSS J0927+2943; see Komossa et al. 2008; Dotti et al. 2009; Bogdanovic et al. 2009), but was subsequently questioned by Decarli et al. (2009a). Boroson \& Lauer (2009) noted that the expected number of chance quasar pairs with projected separations $\lesssim 1^{\prime \prime}$ in the whole SDSS DR7 sample is only $0.3 \%$. On the other hand, if the two objects are physically linked (e.g., if they lie in a rich galaxy cluster), such a number rises significantly (see the discussion in Wrobel \& Laor 2009).

In order to study the properties of this peculiar object, and to probe the presence of a rich galaxy cluster in its environment, we performed deep, high-resolution imaging in the optical and near infrared wavelengths in an ESO Director Discretional Time program (proposal ID: 282.B-5059).

Throughout the paper, we adopt a concordance cosmology with $H_{0}=70 \mathrm{~km} \mathrm{~s}^{-1} \mathrm{Mpc}^{-1}, \Omega_{\mathrm{m}}=0.3, \Omega_{\Lambda}=0.7$.

\section{OBSERVATIONS, DATA REDUCTION, AND ANALYSIS}

$K$-band observations of the field around SDSS J1536+0441 were collected with the High Acuity Wide field $K$-band Imager (HAWK-I) at the ESO/Very Large Telescope (VLT) on 2009 April 30. The total integration time amounts to 48 minutes. HAWK-I is equipped with four CCDs of $2048 \times 2048$ pixels and a pixel scale of $0^{\prime} 1064$ pixel $^{-1}$. Each CCD image $3.2 \times 3.2 \operatorname{arcmin}^{2}$ (after trimming and the removal of partly observed regions). The usual jitter procedure was applied in order to collect broadband images in the NIR. Images were reduced with an $\mathrm{IRAF}^{7}$-based pipeline developed and tested in

\footnotetext{
7 IRAF is distributed by the National Optical Astronomy Observatories, which are operated by the Association of Universities for Research in Astronomy, Inc., under cooperative agreement with the National Science Foundation.
} 


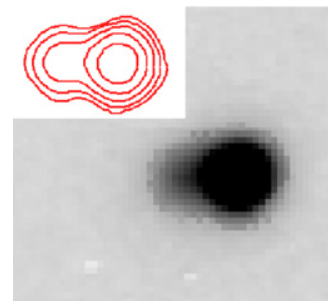

(a)

(b)

(c)

(d)

Figure 1. $K$-band images of SDSS J1536+0441. Each panel is $7^{\prime \prime} \times 6^{\prime \prime}$ wide. North is up, east is to the left. (a) The quasar image, as observed in the $K$ band. The companion galaxy corresponding to VLA-B makes the quasar image clearly oblate toward the left. The contour plots of the radio detection by Wrobel \& Laor (2009) are also shown in the inset, plotted with the same size scale. (b) Image of the companion source, after the subtraction of the quasar model. (c) Image of the quasar, after the subtraction of the model of source B. (d) Residuals after the subtraction of both the models. No obvious asymmetry in the residual map of the two sources is reported, suggesting that the two galaxies are fairly axisymmetric.

other programs (e.g., Kotilainen et al. 2009). The seeing was 0.75-0'.80 (FWHM) throughout the whole integration time. We estimate the zero point by comparing the instrumental magnitudes of field sources with the values reported in the Two Micron All Sky Survey database. The accuracy in the photometric calibration is $0.03-0.05$ mag. Hereafter, we will focus on the Chip 2 image, the one overlapping onto the optical observations.

$V$ - and $z$-band images were collected with the Focal Reducer and low dispersion Spectrograph \#2 (FORS2) at the ESO/VLT on 2009 May 22 and May 24. FORS2 mounts two CCDs of $2048 \times 4096$ pixels. Our observations were carried out with the High-Resolution collimator with $1 \times 1$ binning, yielding a pixel scale of 0 '.0632 $^{\prime}$ pixel $^{-1}$. Offsets within a $10^{\prime \prime}$ wide box were applied in order to overcome the 3 ".5 gap between the two FORS2 CCDs. The final frames are $3.6 \times 3.3 \mathrm{arcmin}^{2}$ wide. In the $V$ band, ten $90 \mathrm{~s}$ long images were acquired for a total integration time of 15 minutes. In the $z$ band, several $120 \mathrm{~s}$ long exposures were secured, yielding a total integration time of 52 minutes. The seeing was excellent $\left(\mathrm{FWHM}=0^{\prime \prime} .7\right.$ in $z, 0^{\prime \prime} .4$ in $V$ ). The zero point of the $z$-band observation was estimated from the $z$ magnitudes of field sources in the SDSS photometric catalog. Similarly, for the $V$ band, we referred to the $V$ magnitude estimates as derived from the SDSS $g$ and $r$ bands, following the conversion recipes by Windhorst et al. (1991). The internal accuracy is around $0.04 \mathrm{mag}$.

A composite image of our data in the three observed bands is available online at http://www.dfm.uninsubria.it/astro/qso_host/ q1536/.

The Astronomical Image Decomposition and Analysis (AIDA; Uslenghi \& Falomo 2008) software is used to model the point-spread function (PSF). The employed technique is widely discussed in Kotilainen et al. (2007, 2009), and it is briefly summarized here. The images of field stars are modeled with the superposition of four bidimensional Gaussians mimicking the

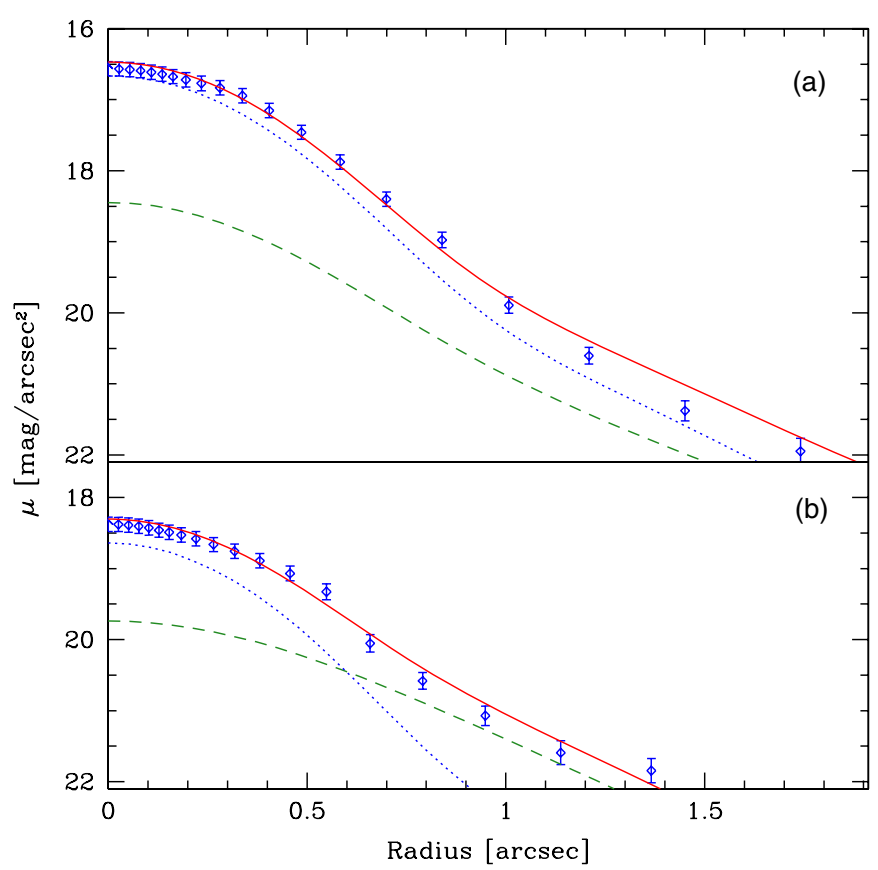

Figure 2. $K$-band light profiles of SDSS J1536+0441 (A) and its companion source (B) as compared to the best-fit model (solid line), the PSF model (dotted line), and the galaxy model (dashed line). The light profile of quasar A shows deviations from the model at large radii, suggesting that the host galaxy may present stellar shells (see Canalizo et al. 2007).

core plus an exponential feature for the wings of the PSF. No significant PSF variation through the frame is reported in the $K$-band frame. On the other hand, FORS2 images are affected by severe distortions of the PSF shape throughout the image, making the PSF model less secure.

\section{RESULTS}

\subsection{Decomposition of the Quasar Image}

Figure 1 shows that the image of SDSS J1536+0441 is clearly elongated eastward, revealing the presence of a blended source (hereafter, source B) close to the quasar and coincident with VLA-B. In a comparison between the PSF model and the observed light profile of the two sources, it is apparent that they are both resolved (see Figure 2). As quasar A is blended with its faint companion, in order to study the presence of extended emission in both of them we adopt an iterative procedure to model the two sources: (1) first, we mask the Eastern side of the bright quasar (where the contribution from the companion source may be relevant) and perform a two-dimensional modeling with a nuclear+host galaxy profile convolved to our PSF model. (2) The model is subtracted from the observed frame, the residuals are masked out and we model the emission from the companion source. (3) The companion model is subtracted from the original observed frame, and we remodel the emission from the quasar. (4) Finally, we re-perform the second step with the new model of the main source.

In order to evaluate the uncertainties in the magnitudes of nuclear and extended emissions, we scaled the Nuclear-to-Host luminosity ratio for a large set of models, and conservatively adopted as reference those bracketing the observed light profiles. Table 1 summarizes the results of the fit procedure. The host galaxy of quasar A is significantly detected only in the $K$ band. On the contrary, source B is well resolved. In all the bands, adding an unresolved nuclear component significantly improves 
Table 1

Best-Fit Parameters in the Modeling of the Image of SDSS J1536+0441

\begin{tabular}{|c|c|c|c|c|c|c|c|c|}
\hline $\begin{array}{l}\text { Band } \\
\text { (1) }\end{array}$ & $\begin{array}{c}m_{\text {tot }} \\
(2)\end{array}$ & $\begin{array}{c}m_{\text {nuc }} \\
(3)\end{array}$ & $\begin{array}{c}m_{\mathrm{gal}} \\
(4)\end{array}$ & $\begin{array}{c}\chi_{\text {nuc }}^{2} / \chi_{\text {gal+nuc }}^{2} \\
(5)\end{array}$ & $\begin{array}{c}\chi_{\mathrm{gal}}^{2} / \chi_{\mathrm{gal}+\mathrm{nuc}}^{2} \\
(6)\end{array}$ & $\begin{array}{c}\text { RF-Band } \\
\text { (7) }\end{array}$ & $\begin{array}{c}M_{\text {nuc }} \\
(8)\end{array}$ & $\begin{array}{c}M_{\mathrm{gal}} \\
(9)\end{array}$ \\
\hline \multicolumn{9}{|c|}{ Source A } \\
\hline$K$ & 14.1 & $14.5 \pm 0.3$ & $15.6 \pm 0.6$ & 1.6 & 3.7 & $H$ & $-26.2 \pm 0.3$ & $-25.3 \pm 0.6$ \\
\hline$z$ & 16.2 & $16.4 \pm 0.2$ & $\sim 18$ & 1.0 & 3.2 & $R$ & $-24.8 \pm 0.2$ & $\sim-23$ \\
\hline$V$ & 17.5 & $17.6 \pm 0.2$ & $\sim 21$ & 1.0 & 4.4 & $B$ & $-23.7 \pm 0.2$ & $\sim-21$ \\
\hline \multicolumn{9}{|c|}{ Source B } \\
\hline$K$ & 15.8 & $16.6 \pm 0.3$ & $16.5 \pm 0.3$ & 2.6 & 1.9 & $H$ & $-24.2 \pm 0.3$ & $-24.6 \pm 0.3$ \\
\hline$z$ & 18.6 & $20.0 \pm 0.3$ & $18.9 \pm 0.3$ & 4.9 & 1.4 & $R$ & $-21.3 \pm 0.3$ & $-22.4 \pm 0.3$ \\
\hline$V$ & 21.0 & $23.5 \pm 0.4$ & $21.2 \pm 0.6$ & 3.6 & 1.3 & $B$ & $-19.0 \pm 0.4$ & $-20.6 \pm 0.6$ \\
\hline
\end{tabular}

Notes. Column 1: band of the observations. Column 2: total apparent magnitude of the model. Column 3: apparent magnitude of the unresolved component. Column 4: apparent magnitude of the host galaxy model. Column 5: comparison between the $\chi^{2}$ obtained assuming a pure PSF and a galaxy+nucleus model. Column 6: the same as Column 5, comparing the cases without and with a nuclear component. Column 7: rest-frame band. Column 8: absolute magnitude of the nucleus in the galaxy+nucleus decomposition. Column 9: the same as Column 8, for the galaxy.

the quality of the fit. As the unresolved-to-resolved luminosity ratio in the $z$ band ( $\approx$ rest-frame $R$ band, see below) is around 0.3 , we cannot assess whether it is a low-luminosity active nucleus (as suggested by the radio detection) or a stellar bulge.

The observed $V, z, K$ bands correspond to the rest-frame $B, R$, and $H$. We compute the absolute magnitudes in these bands as $M_{x}=m_{y}-D M-A_{y}+C_{x-y}$, where $x$ refers to the rest-frame bands $(B, R, H), y$ the observed ones $(V, z, K)$, DM is the distance modulus, $A_{y}$ is the Galactic extinction, and $C_{x-y}$ is a term accounting for filter and $k$-correction. In our cosmological framework, $z=0.3893$ yields $\mathrm{DM}=41.62$. The Galactic extinction is derived from the H I maps by Schlegel et al. (1998). The $k$-correction comes from the galaxy templates by Mannucci et al. (2001) and the quasar template by Vanden Berk et al. (2001). The resulting magnitudes are reported in Table 1. Source A has host galaxy absolute magnitudes consistent with the typical quasar host galaxy luminosities (e.g., Kotilainen et al. 2009) and it is $\sim 2$ mag brighter than the characteristic luminosity of quiescent galaxies at this redshift (Cirasuolo et al. 2008). The companion source is a galaxy $\sim 1 \mathrm{mag}$ fainter (see also the $M_{g}=-21.4$ estimate reported by Lauer \& Boroson 2009) with a much fainter, red nucleus.

We note that, if the $B$-band flux of the unresolved source is interpreted in terms of an AGN, source B should be classified as radio loud, according to the standard definition (e.g., Kellermann et al. 1989) based on the radio-to-optical flux ratio (here, $R \sim 150$, assuming the radio flux found by Wrobel \& Laor 2009).

\subsection{The Properties of the Environment}

In order to investigate the clustering properties of the environment of this quasar, we search for galaxies in its field. We consider the $3.2 \times 3.2 \operatorname{arcmin}^{2}(\approx 1 \times 1 \mathrm{Mpc}$ at $z=0.4)$ region that has been gathered in all the bands. We produce an inventory of sources exceeding a $5 \sigma$ detection threshold with respect to the background count rms through the software SExtractor (Bertin \& Arnouts 1996). The corresponding limit magnitudes are $m_{\mathrm{V}, z, \mathrm{~K}}=25.7,23.1$, and 19.8. We then match the compilations of sources detected in the various bands. The complete list consists of 200 sources detected in the $V$ band, 165 in $z$ (153 of which also appearing in the $V$-band catalog) and 87 in $K$ (80 of which also detected in $V$ ). A total of 69 sources are detected in all the bands.

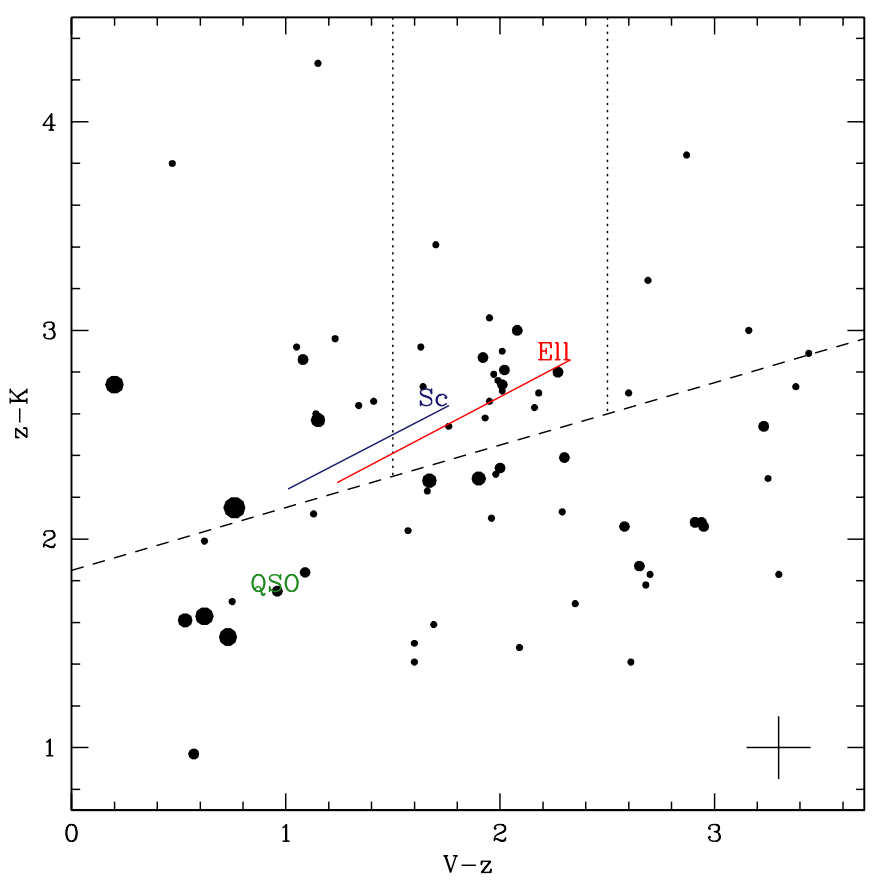

Figure 3. $V z K$ color diagram for the objects in our analysis. Symbol size maps the object brightness. "Ell," "Sc," and "QSO" label the expectation from the elliptical and Sc galaxy templates by Mannucci et al. (2001) and from the quasar template by Vanden Berk et al. (2001), redshifted to $z=0.4$. The solid lines show how galaxy colors change from $z=0$ to $z=0.4$. The typical error box is shown as a cross in the corner of the frame. The color cut in Equation (1) is shown as a dashed line. A number of sources lie within the expectation for galaxies at $z \approx 0.4$ (the vertical, dotted lines), suggesting the presence of a galaxy cluster.

Among them, we select galaxy candidates using the colorcolor diagram proposed by Blanc et al. (2008) and adapted to our photometric system (see Figure 3). We assume that a galaxy has

$$
(z-\mathrm{K})>0.3(\mathrm{~V}-z)+1.85 .
$$

Out of 69 sources, 34 match this color criterion. We note that, given our sensitivity limits, we expect to detect $\sim 30$ stars in our frame in all the bands at this Galactic latitude. ${ }^{8}$ Similarly, if

\footnotetext{
8 Based on the Galaxy simulations performed with the software TRILEGAL
} available at http://stev.oapd.inaf.it/cgi-bin/trilegal (Girardi et al. 2005). 


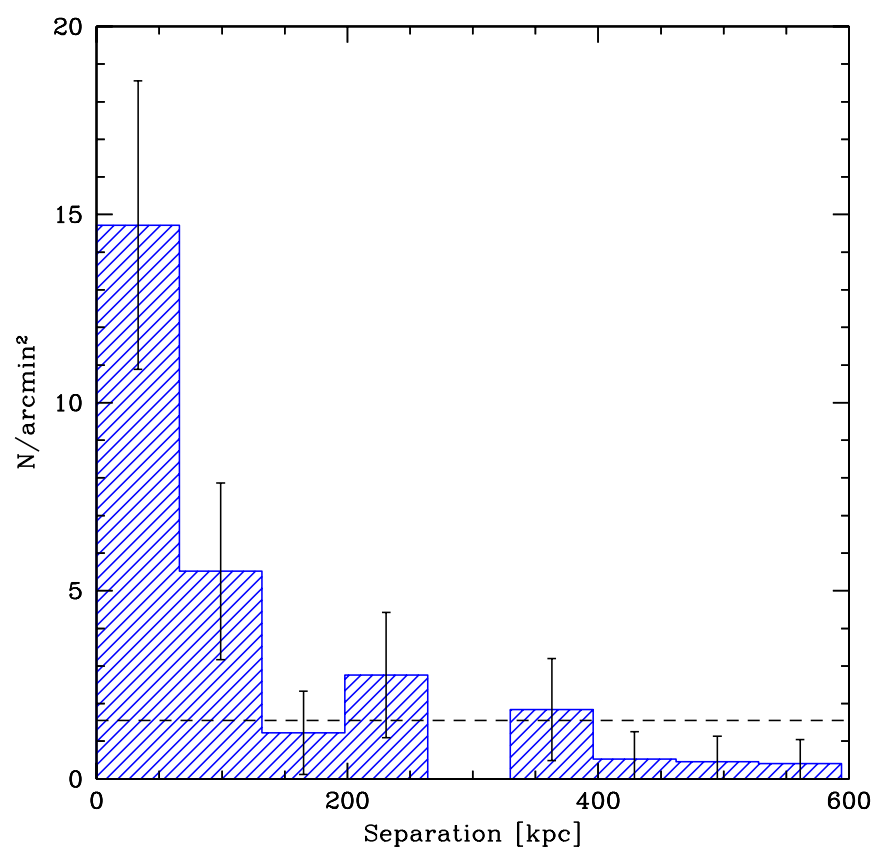

Figure 4. Number density of the galaxy candidates consistent with $z=0.4$ as a function of the projected distance from SDSS J1536+0441. For a comparison, the expected number density of background galaxies is plotted as a dashed line. A clear excess of galaxy candidates within $\sim 200 \mathrm{kpc}$ from the quasar is observed, supporting the cluster scenario.

we consider the number density of general field galaxies (e.g., from Cirasuolo et al. 2008; Blanc et al. 2008), 14 candidates brighter than our sensitivity limits are expected. This indicates that the field around the quasar is overdense. From the galaxy templates by Mannucci et al. (2001), we infer that a $z \approx 0.4$ galaxy has $V-z$ ranging between 1.5 and 2.5 (see also Csabai et al. 2003); thus, 18 out of 34 galaxy candidates are consistent with being at $z \approx 0.4$. The corresponding absolute magnitudes of these objects, assuming that they are galaxies at the same redshift as the quasar, are $\left\langle M_{\mathrm{B}}\right\rangle=-22.8,\left\langle M_{\mathrm{R}}\right\rangle=-20.5$ and $\left\langle M_{\mathrm{H}}\right\rangle=-19.2$ (with $\mathrm{rms} \sim 1 \mathrm{mag}$ ), consistent with typical fairly bright galaxies (e.g., Gavazzi et al. 2003). It is interesting to note that, out of 11 well-resolved sources in the $K$ band, 10 are classified as galaxies in our color selection, and 9 of them are consistent with having $z \approx 0.4$.

We compute the number density of these galaxies as a function of the angular distance from the quasar in order to evaluate the occurrence of any clustering (see Figure 4). We find a significant excess of the galaxy density within $\lesssim 200 \mathrm{kpc}$ from SDSS $\mathrm{J} 1536+0441$ with respect to average field. Thus the quasar is found in a moderately rich cluster of galaxies.

\section{DISCUSSION}

In this Letter, we presented a high-resolution, deep multicolor imaging study of the alleged binary quasar SDSS $\mathrm{J} 1536+0441$. In addition to the extended emission of the host galaxy, we detect a bright companion galaxy at $\sim 1^{\prime \prime}$ from the quasar. The quasar and the companion are located in correspondence with two distinct radio sources observed by Wrobel \& Laor (2009), showing that both galaxies host an AGN.

Our deep images unveil the presence of a rich galaxy cluster. The existence of such a massive structure solves the apparent inconsistency between the observation of two radio sources within $\lesssim 1^{\prime \prime}$ and the expected number of such pairs in the SDSS catalog, as discussed in Boroson \& Lauer (2009) and Wrobel \& Laor (2009). Furthermore, the potential well of such a rich galaxy cluster could, in principle, explain the shift in redshift between the two sets of emission lines. This consideration favors a superposition picture to explain the spectrum of SDSS $\mathrm{J} 1536+0441$.

However, the red color and the faintness of the nuclear optical continuum observed in the companion galaxy suggest that its AGN emission is obscured. As a consequence, the AGN of the companion galaxy should not contribute much to the broad emission lines present in the SDSS spectrum, in agreement with the indications discussed in Chornock et al. (2009b) and Lauer \& Boroson (2009). In this case, the two sets of broad emission lines should be emitted by the quasar, consistently with the BHB or the double peaked emitter scenarios. The external regions of the companion galaxy could also naturally explain the narrow absorption lines observed in the spectrum of SDSS $\mathrm{J} 1536+0441$, as already suggested by Lauer \& Boroson (2009). The origin of the red bump in the broad line profiles reported by Chornock et al. (2009b) remains unclear both in the BHB and the superposition scenarios.

The effective role of the companion in the spectrum of SDSS $\mathrm{J} 1536+0441$ and of the surrounding galaxy cluster is yet to be clarified. The superposition model, where the companion emits one of the two sets of emission lines is in possible conflict with the presently available spectroscopic data. However, the discovery of a possible nucleus in source B, matched with its radio detection by Wrobel \& Laor (2009), may support this picture. Crucial tests for this interpretation can be derived: from UV observations of high-ionization lines (as discussed in Chornock et al. 2009b), high angular resolution optical spectroscopy and high-resolution X-ray images.

This research has made use of the NASA/IPAC Extragalactic Database (NED) which is operated by the Jet Propulsion Laboratory, California Institute of Technology, under contract with the National Aeronautics and Space Administration.

Facilities: VLT(FORS2) VLT(HAWK-I)

\section{REFERENCES}

Bertin, E., \& Arnouts, S. 1996, A\&AS, 117, 393

Bogdanović, T., Eracleous, M., \& Sigurdsson, S. 2009, ApJ, 697, 288

Boroson, T. A., \& Lauer, T. R. 2009, Nature, 458, 53

Blanc, G. A., et al. 2008, ApJ, 681, 1099

Canalizo, G., Bennert, N., Jungwiert, B., Stockton, A., Schweizer, F., Lacy, M., \& Peng, C. 2007, ApJ, 669, 801

Chornock, R., et al. 2009a, ATel, 1955, 1

Chornock, R., et al. 2009b, ApJL, submitted (arXiv:0906.0849)

Cirasuolo, M., et al. 2008, MNRAS, submitted (arXiv:0804.3471)

Csabai, I., et al. 2003, AJ, 125, 580

Decarli, R., Reynolds, M. T., \& Dotti, M. 2009a, MNRAS, 397, 458

Decarli, R., Treves, A., Falomo, R., Dotti, M., Colpi, M., \& Kotilainen, J. K. 2006b, ATel, 2061, 1

Dotti, M., Montuori, C., Decarli, R., Volonteri, M., Colpi, M., \& Haardt, F. 2009, MNRAS, 398, L73

Gavazzi, G., Boselli, A., Donati, A., Franzetti, P., \& Scodeggio, M. 2003, A\&A, 400,451

Girardi, L., Groenewegen, M. A. T., Hatziminaoglou, E., \& da Costa, L. 2005, A\&A, 436, 895

Heckman, T. M., Krolik, J. H., Moran, S. M., Schnittman, J., \& Gezari, S. 2009, ApJ, 695, 363

Kellermann, K. I., Sramek, R., Schmidt, M., Shaffer, D. B., \& Green, R. 1989, AJ, 98, 1195

Komossa, S., Zhou, H., \& Lu, H. 2008, ApJ, 678, L81 
Kotilainen, J. C., Falomo, R., Decarli, R., Treves, A., Uslenghi, M., \& Scarpa, R. 2009, ApJ, in press

Kotilainen, J. K., Falomo, R., Labita, M., Treves, A., \& Uslenghi, M. 2007, ApJ, 660, 1039

Lauer, T. R., \& Boroson, T. A. 2009, ApJL, in press

Mannucci, F., et al. 2001, MNRAS, 326, 745
Schlegel, D. J., Finkbeiner, D. P., \& Davis, M. 1998, ApJ, 500, 525

Uslenghi, M., \& Falomo, R. 2008, Proc. Erice, in press

Vanden Berk, D. E., Richards, G. T., Bauer, A., Strauss, M. A., Schneider, D. P.,

Heckman, T. M., York, D. G., \& Hall, P. B. 2001, AJ, 122, 549

Windhorst, R. W., et al. 1991, ApJ, 380, 362

Wrobel, J. M., \& Laor, A. 2009, ApJ, 699, L22 